\title{
PRECISE RADAR TARGET RANGING ESTIMATION BASED ON SLIP MATCHING OF ENVELOPES
}

\author{
Liqiang Zhu, Yongxiang Liu*, Dekang Zhu \\ School of Electronic Science and Engineering, National University of Defense Technology, Changsha, \\ China \\ 909668383@qq.com, lyx_bible@sina.com, dekang.zhu@foxmail.com, *the corresponding author is \\ Yongxiang Liu
}

\begin{abstract}
A novel precise radar target ranging method based on echo phases and slip matching of envelopes is presented in this paper. The method provides a high precision with rootmean-square error at sub-wavelength level, and it only requires envelopes of the echo pulses. The difficulty is matching procedure of envelopes. According to the principle of resolving ambiguous phases based on slip matching of envelopes, some restraint conditions for ambiguity resolution are proposed, and the theory of extracting ambiguous Doppler phases from wideband radar echoes is provided. The method provides a more convenient and precise way to resolve ambiguous phases, and the experiments have validated the performance of ranging capability.
\end{abstract}

Index Terms - micro-motion, phase derived range, slip matching of envelopes, ambiguity resolution.

\section{INTRODUCTION}

Micro-motion is the mechanical dynamics of a target or any structure on the target in addition to bulk motion, including rotation, vibration, tumbling, precession and so on ${ }^{[1]}$. Characteristic analysis of radar target with micro-motions have gotten more and more attentions in radar community ${ }^{[2]}$.

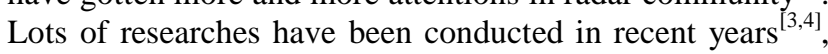
such as imaging of radar target with micro-motions, modulation mechanics of micro-motions on electromagnetic wave, extraction of micro-motions' feature, estimation of micro-motions parameters, and so on. The key technical challenge among these researches is micro-motion characteristic acquisition because of its weakness in radar echoes, especially under the conditions such as small amplitude of micro-motions, low working frequency of radar, or inaccurate compensation of bulk translation ${ }^{[5]}$.

The precise estimation of radar target range is an effective way to solve the micro-motion characteristic acquisition problem. It has been reported that the X-band radar GBR and SBR in the missile defense systems of the USA have a highly precise measurement capability of range and velocity ${ }^{[6]}$. The key technology is phase derived range (PDR), which has highly precise measurement capability of range at sub-wavelength level. And it guarantees higher data rate and discriminates more dynamics details of target compared with the traditional Doppler method. There are two technical cores in PDR, which are the phase extraction from wideband radar echo and resolution of ambiguous Doppler phase considering different radar conditions. As far as the resolution of ambiguous phase is concerned, the traditional method is integrating Doppler frequency to resolve the ambiguous phase. But it needs precise phase measurement value, which radars sometimes can not provide.

In this paper, a novel method of resolving ambiguous phase based on slip matching of envelopes is presented. The works are organized as follows: the basic principle of resolving ambiguous phase based on slip matching of envelopes is analyzed in Section 2, and the restraint conditions for the method is considered and analyzed in section 3. In section 4, the extraction of ambiguous Doppler phase is developed. The experimental results are presented in Section 5, showing the performance of the method on ambiguity resolution and measurement of the range.

\section{RESOLVE AMBIGUOUS PHASE BASED ON SLIP MATCHING OF ENVELOPES}

For one-dimensional range profiles of target, the peaks of envelopes represent target scatterers. By observing the trail of the envelope, the target physical movement information can be obtained. In order to get the number of range bins between successive profiles, the correlation function is utilized and it goes the max when the two successive envelopes match the most. Vary the slip ranging bins with step by one range bin, and figure out how many range bins have been slid when the result of correlation function is maximal. After obtaining the range bins between the two successive envelopes, the corresponding ambiguous circles $\mathrm{K}$ can be estimated accurately. The resolution of ambiguous Doppler phase based on slip matching of envelopes can be realized, as shown in Fig. 1. 


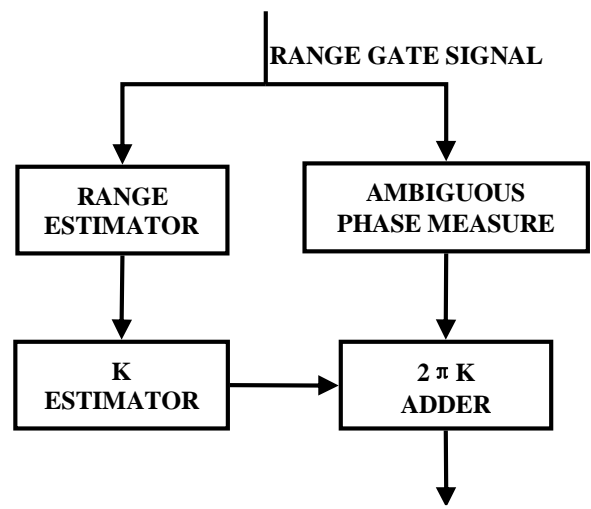

UNAMBIGUOUS DOPPLER PHASE

Fig. 1. Procedure of resolving ambiguous phase

After receiving the radar echoes, the ambiguous circles and ambiguous phase can be yielded soon. The phase can be translated to the range with error values at sub-wavelength level. According to the theory of phase derived range, the relationship between radial range and Doppler phase can be expressed as

$$
R=\frac{c \Delta \phi}{4 \pi f_{0}}=\frac{\lambda}{4 \pi} \Delta \phi
$$

where $\Delta \phi$ denotes unambiguous Doppler phase, $R$ denotes radial range and $f_{0}$ is the carrier frequency of radar.

The sketch map of slip matching of envelopes is shown in Fig.2.

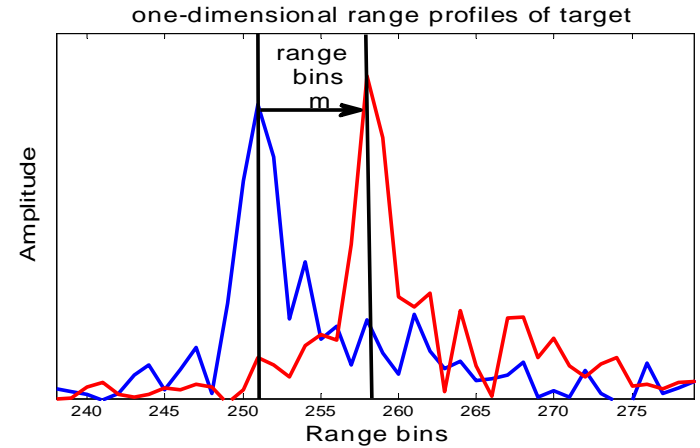

Fig.2. Sketch map of envelope's slip match

As observed in Fig.2, the horizontal axis is divided by range bin $\rho_{r}=c / 2 B$ and target moves in one interval of pulse. Counting how many range bins have been slid when the peak of the envelope matches the previous one the most, the radial distance with large error values can be yielded $r=m \bullet c / 2 B, m$ denotes the number of range bins between the two envelopes. It is an integer. And the ambiguous circles can be obtained from $r$.

$$
r=\lambda \cdot K / 2+r_{0}
$$

where $K$ denotes the ambiguous circles, and $r_{0}$ represents radial range corresponding to the ambiguous phase and it is inaccurate. But the ambiguous circles $K$ is accurate.

$$
K=r \bmod (\lambda / 2)
$$

The unambiguous Doppler phase can be yielded with $\mathrm{K}$.

$$
\theta_{d}=\theta_{a}+2 K \pi
$$

The Doppler radial range is calculated as follows:

$$
R=\frac{\theta_{d} \lambda}{4 \pi}
$$

For complex target, there are many scatterers. In addition to bulk motion, target sometimes has micro-motion with large amplitude, resulting in the change of scatterers' relative location and mix of envelopes from different range window. In this condition, method proposed in this paper is no longer useful. So target is assumed not to have micromotion with large amplitude or mechanical dynamics which can change the relative location of target scatterers such as tumbling.

\section{RESTRAINT CONDITIONS FOR SLIP MATCHING OF ENVELOPES}

As mentioned in section 1, there are some restraint conditions for slip matching of envelopes. The parameters and measurement error values of radar mainly influence the validity of this method. Let $\bar{D}_{m}$ be the estimated value of Doppler phase increment $D_{m}$ and $\widetilde{D}_{m}$ be the measurement value. They can expressed as

$$
\left\{\begin{array}{l}
\bar{D}_{m}=D_{m}+\delta \bar{D}_{m} \\
\widetilde{D}_{m}=D_{m}+\delta \widetilde{D}_{m}
\end{array}\right.
$$

where $\delta \bar{D}_{m}$ denotes the error of $\bar{D}_{m}$ and $\delta \widetilde{D}_{m}$ denotes the error of $\widetilde{D}_{m}$. There's a necessary condition to resolve the ambiguous phase correctly.

$$
\left|\delta \bar{D}_{m}-\delta \tilde{D}_{m}\right|<\pi
$$

But in this condition, it does not reflect the restraint relationship between the resolution of the ambiguous phase and the parameters of the radar measurements directly.

Using aforementioned equations, $\bar{D}_{m}$ can be yielded

$$
\bar{D}_{m}=2 \pi\left\{\left(m \cdot \frac{c}{2 B}\right) \bmod \left(\frac{\lambda}{2}\right)\right\}+\theta_{a}
$$

So, the $\delta \bar{D}_{m}$ can be written as

$$
\delta \bar{D}_{m}=2 \pi\left\{\left(m^{\prime} \cdot \frac{c}{2 B}\right) \bmod \left(\frac{\lambda}{2}\right)\right\}+\theta_{a}{ }^{\prime}
$$

where $m^{\prime}$ is the error value of range bins envelope has been slide, $\theta_{a}{ }^{\prime}$ is the error value of ambiguous phase.

Considering $\delta \widetilde{D}=\varepsilon_{m+1}-\varepsilon_{m}$, let $\sigma_{D}$ be the root-meansquare-error of error value of radar's phase measurement error, so $\delta \widetilde{D} \sim N\left(0,2 \sigma_{D}^{2}\right)$. According to the principle of $3 \sigma$, $\delta \widetilde{D}<3 \sqrt{2} \sigma_{D}$ can be gotten. Referencing (7) and (9), we finally obtain the relationship betweenn error value of measurement phase, bandwidth of radar and the error value of range bins. 


$$
\left\{\left(m^{\prime} \cdot \frac{c}{2 B}\right) \bmod \left(\frac{\lambda}{2}\right)\right\}<\frac{\pi-3 \sqrt{2} \sigma_{D}-\theta_{a}{ }^{\prime}}{2 \pi}
$$

\section{EXTRACT OF AMBIGUOUS DOPPLER PHASE}

A linear frequency modulation(LFM) signal has been chosen as the transmitted signal. The $m$ th transmitted pulse is

$$
s_{t}(t)=\operatorname{rect}\left(\frac{\hat{t}}{T}\right) \exp \left[j 2 \pi\left(f_{0} t+\frac{1}{2} \gamma^{2} \hat{t}^{2}\right)\right]
$$

where $\hat{t}$ denotes fast-time ${ }^{[7]}$, slow time is $t_{m}=m \cdot \operatorname{Tr}$, and $t=t_{m}+\hat{t}, f_{0}$ is the carrier frequency of the transmitted wave, $\gamma$ denotes the slope of frequency modulation. Target is assumed to move at a constant velocity at radial direction, then the target radial range is

$$
R(t)=R\left(t_{m}\right)+v \hat{t}
$$

$R\left(t_{m}\right)$ denotes the radial range between target and radar when $t=t_{m}$, and the corresponding time delay is

$$
\tau^{\prime}(t)=\frac{2 R(t)}{c}=\frac{2 \cdot\left(R\left(t_{m}\right)+v \hat{t}\right)}{c}=\tau\left(t_{m}\right)+\frac{2 v \hat{t}}{c}
$$

where $\tau\left(t_{m}\right)$ denotes the corresponding time delay that the $m$ th transmitted pulse returns from target, briefing as $\tau$, and the corresponding echo is ${ }^{[8,9]}$

$$
\begin{aligned}
& S_{r}(t, \hat{t})=\operatorname{rect}\left(\frac{\left(1-\frac{2 v}{c}\right) \hat{t}-\tau}{T}\right) \\
& \cdot \exp \left\{j 2 \pi\left[f_{0}\left(t-\tau-\frac{2 v \hat{t}}{c}\right)+\frac{1}{2} \gamma\left(\left(1-\frac{2 v}{c}\right) \hat{t}-\tau\right)^{2}\right]\right\}
\end{aligned}
$$

After the dechirping process the echo yields

$$
\begin{aligned}
S_{i f}\left(\tilde{t}, t_{m}\right) & =\operatorname{rect}\left[\frac{\tilde{t}-\left(\frac{2 R_{\Delta}}{c}+\frac{2 v}{c} \tau_{0}\right)}{T}\right] \\
& \cdot \exp \left\{-j 2 \pi\left[\varphi_{1}+\varphi_{2}+\varphi_{3} \tilde{t}+\varphi_{4} \tilde{t}^{2}\right]\right\}
\end{aligned}
$$

where $R_{\Delta}=\frac{c}{2}\left(\tau-\tau_{0}\right)$,

$$
\left.\begin{array}{ll}
\varphi_{1}=-\gamma\left(\frac{2 R_{\Delta}^{2}}{c^{2}}+\frac{4 v}{c^{2}} R_{\Delta} \tau_{0}\right) & \varphi_{2}=f_{0}\left(\frac{2 R_{\Delta}}{c}+\frac{2 v}{c} \tau_{0}\right) \\
\varphi_{3}=\gamma\left(\frac{2 R_{\Delta}}{c}+\frac{2 v}{c} \tau_{0}\right)+f_{0} \frac{2 v}{c} & \varphi_{4}=\gamma \frac{2 v}{c}
\end{array}\right\}
$$

After fast method of dechirping and phase compensation, the echo finally yields

$$
S\left(\tau, t_{m}\right)=T \exp \left\{-j 2 \pi f_{0} \tau\right\}
$$

where $f_{0} \tau$ is the ambiguous Doppler phase.

\section{EXPERIMENTS RESULTS}

In this section, experiments intend to validate the algorithm performance using radar measurement data of cone target with procession, including the extraction of Doppler phase from wideband radar and resolution of ambiguous phase. The procession is a typical kind of micro-motion for space target. A metal cone target is designed to conduct procession, the length is $1.4 \mathrm{~m}$, the diameter of bottom is $0.37 \mathrm{~m}$, as shown in Fig.3. There are three engines for driving the cone in precession with the variable parameters such as the angle of precession $\alpha$, the cycle of precession T, and the angle $\theta$ between line of radar sight and precession axis. Radar works with sweep frequency mode, operating at $\mathrm{X}$ band (9.5$10.5 \mathrm{GHz}$, the bandwidth is $1 \mathrm{GHz}$ and the frequency step is $10 \mathrm{MHz})$ and $\mathrm{K}$ band $(14.5-16 \mathrm{GHz}$, the bandwidth is $1.5 \mathrm{GHz}$, and the frequency step is $15 \mathrm{MHz}$ ), the pulse width is $100 \mathrm{~ms}$, and the pulse repetition time is $200 \mathrm{~ms}$.

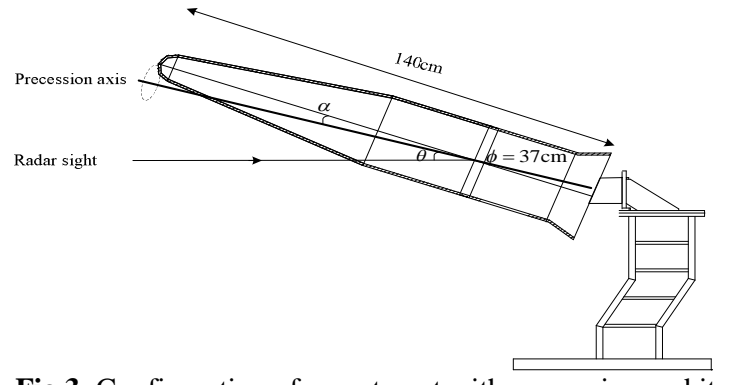

Fig.3. Configuration of cone target with precession and its radar measurement

In order to acquire the characteristic of micro-motions with the proposed method above, the first step is to resolve the different scatterers in the target by the sequences of 1-D HRRP, the second step is to extract the Doppler phase from peaks in HRRPs, and the final step is to determine the relative radial range of corresponding parts of cone based on phase resolution with the scatterers association. Fig.4 shows three successive sequences of 1-D HRRP of target with bulk motion, and the velocity $=25 \mathrm{~m} / \mathrm{s}$. The horizontal axis represents radial range, while the vertical one represents the amplitude.

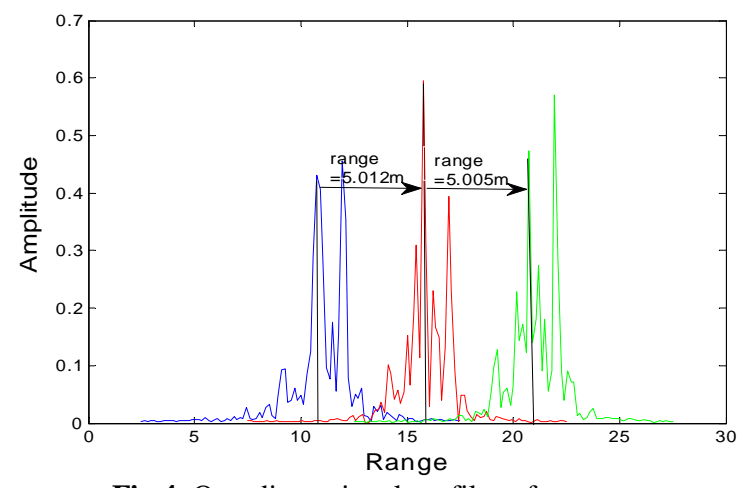

Fig.4. One-dimensional profiles of target 
Fig.5(a) shows the relative radial range of scatterers on the target with precession of $\mathrm{K}$ band $(14.5 \mathrm{GHz})$, where the initial value of every scatter radial range is zero, indicating that the position of scatter at the instant of the first pulse is considered as reference zero position, with the parameters of $\alpha=5^{\circ}, T=10 \mathrm{~s}, \theta=0^{\circ}$. Fig.5(b) is measured at $\mathrm{X}$ band with bandwidth of $1 \mathrm{GHz}$, with the parameters of $\alpha=5^{\circ}$, $T=10 \mathrm{~s}, \theta=25^{\circ}$. The precession is assumed to be a sine signal, whose amplitude is $0.0075 \mathrm{~m}$ and frequency is $0.1 \mathrm{~Hz}$ in figure.5(a), and $0.035 \mathrm{~m}$ in amplitude and $0.1 \mathrm{~Hz}$ in frequency in figure.5(b). Because of the measurement error and mismatch of slip matching procedure, the error value of radial range is varying as an abnormal sine signal. But the amplitude and frequency can be estimated from the figure accurately.

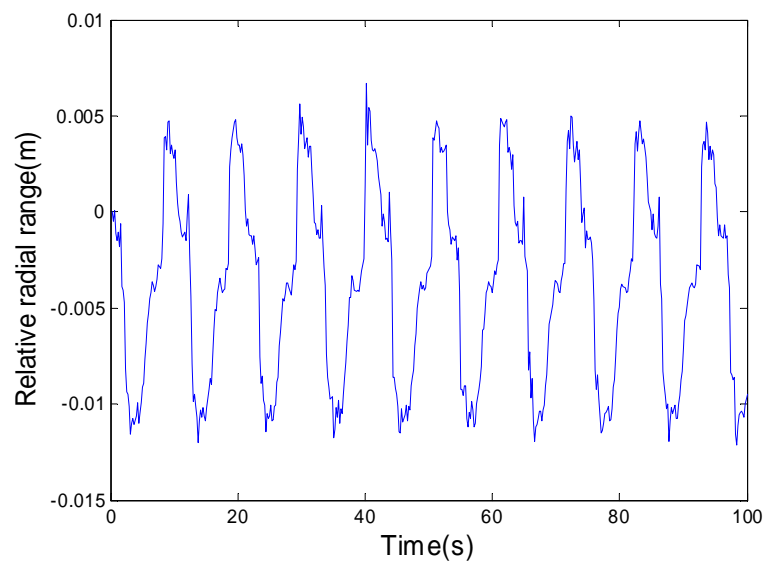

(a) Measured at $\mathrm{K}$ band with bandwidth of $1.5 \mathrm{GHz}$

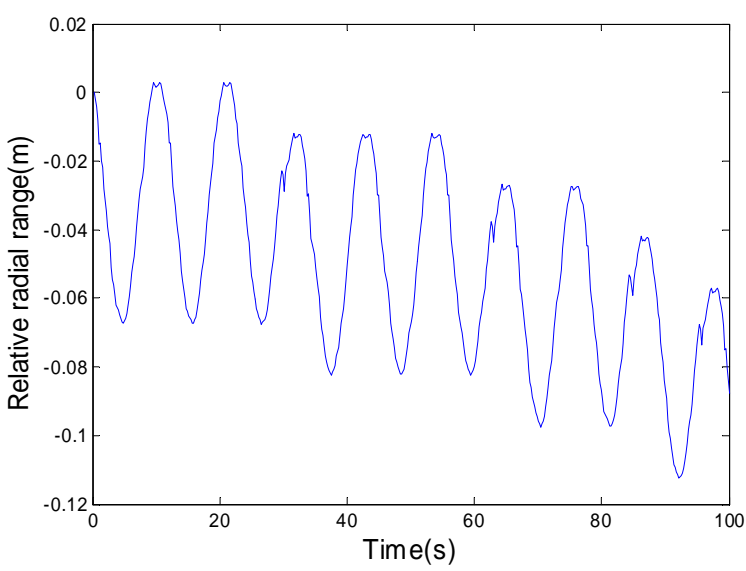

(b) Measured at $\mathrm{X}$ band with bandwidth of $1 \mathrm{GHz}$

Fig.5. Relative radial range of scatter on the target

The results have demonstrated that proposed method can provide the radial range with high precision at the level of sub-wavelength. The results also have shown the feasibility of resolving ambiguous phase by slip matching of envelopes.

\section{CONCLUSION}

A novel method of resolving ambiguity in phase derived range based on slip matching of envelopes is presented in this paper. This algorithm has two advantages. First, slip matching of envelopes algorithm has a high precision with error at sub-wavelength level. Second, comparing to traditional integral of Doppler frequency algorithm, this method only needs envelopes, which provides a more convenient way to resolve ambiguous phase.

However, as mentioned above, this algorithm needs a relatively simple structure of targets and may be invalid in the condition of scatterers' relative location variations. The method of slip matching of envelopes still needs further researches.

\section{ACKNOWLEDGEMENT}

The work in this paper has been supported by the National Science Fund of China under Grant No.61422114, and Natural Science Fund for Distinguished Young Scholars of Hunan Province under Grant No.2015JJ1003

\section{REFERENCES}

[1] V. C. Chen, F. Y. Li, S. S. Ho, and H. Wechsler. MicroDoppler effect in radar: phenomenon, model, and simulation study[J]. IEEE Transactions on Aerospace and Electronic systems, vol. 42, no.1, pp2-21, Jan. 2006.

[2] V. C. Chen. The micro-Doppler effect in radar [M]. Boston: Artech House, 2011.

[3] Y. X. Liu, K. L. Li, X. Li, Z. W. Zhuang. Characteristic Analysis of Radar Target with Micro-motion[J]. Science and Technology Review, vol. 29, no. 22, pp 7279, Aug. 2011.

[4] Y. X. Liu, D. K. Zhu, Z. W. Zhuang. Micromotion Characteristic Acquisition Based on Wideband Radar Phase[J]. IEEE transactions on geoscience and remote sening, vol. 52, NO. 6, pp50-57, june. 2014.

[5] J. S. Ma. The Measure Ability and the parameters of NMD-GBR radar[J]. Aerospace Electronic Warfare, 2002: $1-8$

[6] Zheng Bao, Mendao Xing, Tong Wang. "The Technique of Radar Image" $[\mathrm{M}]$. Publishing House of Electronics Industry, Beijing, 2005: 132-134.

[7] D. K. Zhu, Y. X. Liu, L. K. Le, X. Li. Feature Extraction for Target with Micro-Motion Based on Radar Phase Derived Range. Journal of Astronautics[J], vol.34, No.4, pp74-81, April 2013.

[8] D. J. Feng. Phase Signatures and Compensating Approach to Moving Target Echoes by Dechirping Processing[J]. Journal of Electronics and Information Technology, vol. 30, no.4, pp16-20, Apr. 2008.

[9] D. J. Feng. Method of Measuring Range and Velocity of Space Target Based on Single Wideband Pulse[J]. Journal of Signal Processing, vol. 22, no.1, pp73-77, Feb. 2006. 